\title{
MATHEMATICAL MODELLING OF FIBRE WINDING PROCESS FOR COMPOSITE FRAMES
}

This article describes the authors' own mathematical modelling designed for the production process of a new type of low-weight composite frame. The used real technology is based on the winding of carbon or glass filament rovings around a polyurethane core which is a frame with a circular cross section (this type of composites is used, for example, to reinforce the doors and windows of airplanes). The core is attached to the end-effector of the robot (robot-end-effector) and successively passes through the fibre-processing head during the winding process. Quality production depends primarily on the correct winding of fibres around the polyurethane core. It is especially needed to ensure the correct angles of the fibre winding around the polyurethane core and the homogeneity of individual winding layers. The numerical model described in Euclidean space $E 3$ of the manufacturing process is used when the fibre-processing frame is passing through the fibre-processing head. We use the described mathematical model and matrix calculus to enumerate the trajectory of the robot-end-effector to determine the desired passage of the core through the fibre-processing head. The calculated sequence of "tool-centre-point" values of the robot allows us to define the desired trajectory of the robot-end-effector and, thereby, the passage of the frame through the fibre-processing head. The calculation of the trajectory was programmed in the Delphi development environment. A practical example is analysed in the article.

Keywords: Mathematical modelling, composite frame, winding fibre processing, robot technology.

\section{Introduction}

This article describes the authors' own mathematical modelling for the production process of a new type of low-weight composite frame. A new type of low-weight frame composite of high quality is used for example in the transport industry [1, 2 and 3]. Reasons for the use of composites not only in transport industry (car industry, aerospace industry, aeronautic industry, etc.) are derived from the current requirements for the low-weight parts. The construction of composite frames must be light because, for example, in car industry an optimal weight and power usage lead to emission reductions. But concurrently the construction of design parts must be sufficiently solid and rigid. We will describe technology of carbon or glass filament rovings wound around the polyurethane core which is a frame shape in $3 \mathrm{D}$ with a varying shape and size of cross-section of the frame. In the following part of the article we will consider only a circular cross-section. If the frame cross-section is not circular, we consider an imaginary cylindrical "envelope" (with minimum possible radius) stretched on the frame surface. Traditional procedures of composite manufacturing are labour- intensive and time-consuming. Moreover, the traditional techniques do not ensure accurate fibre winding around the core. The use of industrial robots in composite production greatly reduces production costs, production time and minimises scrap rate. Other problems of industrial robot trajectory are also solved in publications, e.g. [4 7]. Composites offer an attractive material properties-to-production cost ratio. One of the possible methods for producing composites is to stretch the fabric from the fibres on a core with an arbitrary geometry. However, if the core of the composite is a 3D frame or a frame with a very complicated 3D shape or several layers of the fibre strands are wound simultaneously around the core, then this method is not suitable. In such cases, the method of dry winding of endless fibre strands around a core geometry using a rotary fibreprocessing head is often used for the composite production. This method provides full control over the placement, laying direction, and the amount of fibres on the core as well as the homogeneity of the structure. The final composite is obtained after dry winding of the required layers of strands around the core by injection of the resin to the mould using heat and pressure. Now, we are describing the process of producing composites with a polyurethane core of

\footnotetext{
* ${ }^{1}$ Michal Petru, ${ }^{2}$ Jaroslav Mlynek, ${ }^{1}$ Tomas Martinec, ${ }^{3}$ Jozef Broncek

${ }^{1}$ The Institute for Nanomaterials, Advanced Technologies and Innovation, Technical University of Liberec, Czech Republic

${ }^{2}$ Department of Mathematics and Didactics of Mathematics, Faculty of Science, Humanities and Education, Technical University of Liberec, Czech Republic

${ }^{4}$ Department of Design and Mechanical Elements, Faculty of Mechanical Engineering, University of Zilina, Slovakia

E-mail: michal.petru@tul.cz
} 
a frame shape by the method of dry winding around the core. We tested this manufacturing process in our experimental laboratory (Fig. 1). The key equipment in our experimental laboratory is an industry robot KR 16-2 and fibre-processing head as shown in Fig. 2.

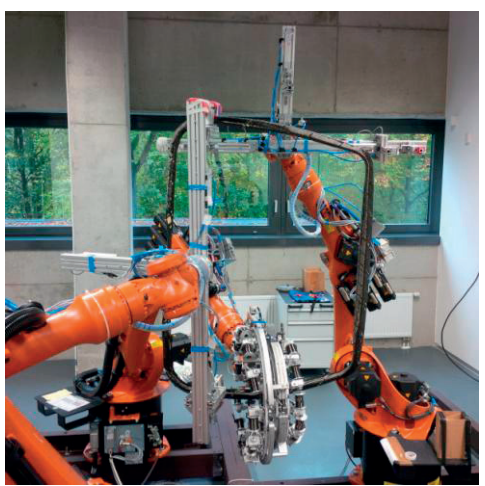

Fig.1 Experimental laboratory for manufacturing process of winding fibres around the frame
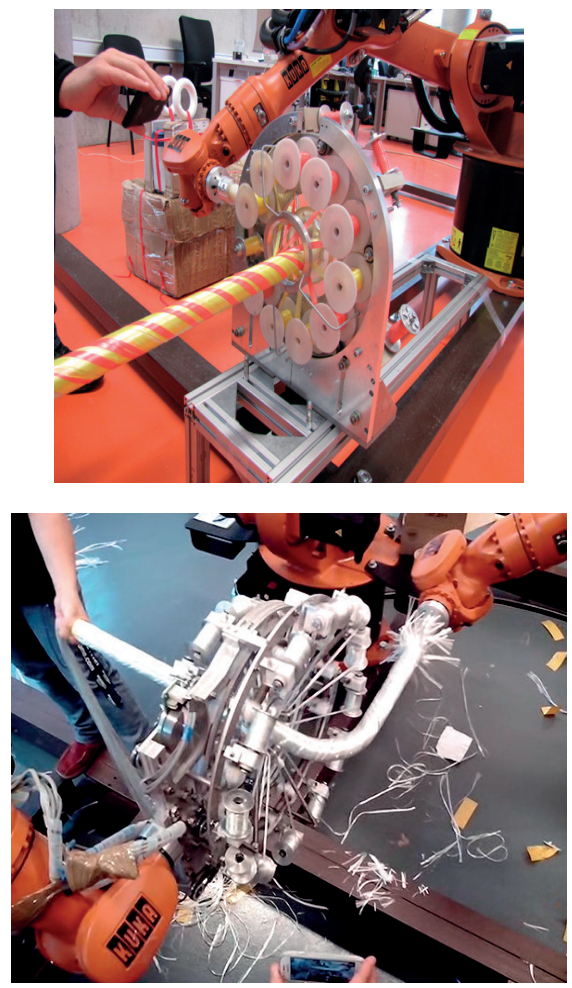

Fig.2 Fibre-processing head with one guide line (up) and with three guide lines on the frame (down)

The fibre-processing head is fixedly placed in the workspace of the robot and coordinates of its parts are specified in the basic coordinate system of the robot. The used fibre-processing head contains three guide lines (Fig. 2). Each guide line contains twelve fixed fibre coils along its periphery. The outer guidelines rotate around a common axis and the intermediate guideline is static. The polyurethane core of a frame shape is attached to the robot- end-effector. The passage of the core through the fibre-processing head is controlled by the movement of the robot-end-effector. When polyurethane core passes through fibre-processing head the strands are successively wound on the surface of the core at a targeted angle. First, the outer rotating guide line ensures winding strands under the angle $45^{\circ}$ (relative to the axis of the head and the moving direction of the core). Subsequently, the middle static guide winds the second layer of strand under the angle $0^{\circ}$ and the second outer rotation winds the final layer of strands $-45^{\circ}$. Our goal is for the core to pass through the fibreprocessing head orthogonal to the guide lines of the head as far as possible. The principle of the winding solution is shown in Fig. 2 (fibre processing head contains only one rotation line in this figure). To optimise the manufacturing process we made a numerical model for calculating the robot-end-effector trajectory to achieve an optimal directional orientation of the fibre placement in the manufacture of the composite frame profile. The mathematical calculation of trajectory was programmed in the Delphi development environment. This article describes a numerical model for quality of the manufacturing process technology of a shaped composite in 3D space. As already mentioned, providing the correct angles for winding of strands around the core is mainly conditioned by the determination of the appropriate trajectory of the robot-end-effector. Orthogonal direction of passage of the core (of a closed frame shape) to the guide line in the place of its own winding of fibres around the core surface ensures the correct angle and uniformity of winding. The quality of fibre winding also depends on the material properties of polyurethane core and fibres (especially on adhesion of the fibre to the core).

\section{Numerical model describing the fibre winding process for a composite frame}

This chapter briefly describes the numerical model of the optimal process of winding strands around a 3D polyurethane core with a circular cross-section. The core shape is a frame and the radius of the circular cross section of the frame may be generally different for individual parts of a frame. The actual process of strand winding is carried out using a fibre-processing head and an industrial robot. The polyurethane core is attached to the robot-end-effector and the winding head is fixed in the workspace of the robot. Within the described numerical model (Fig. 3), we will consider the right-handed Euclidean coordinate system $\mathrm{E}_{3}$ of the robot $(B C S)$. We will describe the positions of the individual parts of an experimental workplace of winding strands to frame using this coordinate system. Subsequently, we will consider the local right-handed Euclidean coordinate system of the robot-end-effector $(L C S)$. To avoid any misunderstanding, we will label the points and vectors with coordinates in $B C S$ with the subscript $B C S$ and points and vectors with coordinates in 
$L C S$ with the subscript $L C S$. Activities of the industrial robot are controlled using a robot control unit (in our case unit KR C4) and the library of instructions. For our purposes it is crucial to set the desired position of the robot-end-effector using the library of instructions. The position and orientation of the robot-endeffector is defined by using the LCS. The origin of the LCS is positioned in the robot-end-effector while at the same time the robot-end-effector is oriented in the direction of the positive part of the $z$-axis in the $L C S$ with regard to the $B C S$. The actual position of the $L C S$ with regard to the $B C S$ is determined by the values listed in the "tool-centre-point" $(T C P)$. The tool centre point contains six values $T C P=(x, y, z, a, b, c)$. The first three parameters specify the coordinates of the origin of the $L C S$ with regard to the $B C S$. The values $a, b$ and $c$ indicate the angle of the rotation of the LCS around the axes $z, y$ and $x$ with regard to the $B C S$. The non-bearing polyurethane frame can be described by the central axis $o$ (see Fig. 4) and radius $r_{\text {TUBE }}$ of the cross-section of the frame. The central axis of the frame $o$ is entered in the LCS of the robot-end-effector using a discrete set of $N$ points stored in the array $B: a r r a y[1 . . N, 1 . .3]$ where $B[i, 1]$ indicates the $x, B[i, 2]$ indicates the $y$ and $B[i, 3]$ indicates the $z$ coordinate of the $i$-th point $B[i]_{L C S}$ lying on the axis $o$ (see Fig. 4). At the same time, the array vector $1 B$ :array $[1 . . N, 1 . .3]$ is entered where vector $1 B$ :array $[i, 1]$, vector $1 B: \operatorname{array}[i, 2]$ and vector $1 B: \operatorname{array}[i, 3]$ indicate the coordinates of the unit vector vector $1 B$ :array $[i]_{L C S}$ tangent to the axis $o$ at point $B[i]_{L C S}$. In addition, the array vector $2 B[i, 1]$ is defined where, vector $2 B[i, 2]$ and vector $2 B[i, 3]$ indicate the coordinates of the unit vector vector $2 B[i]_{L C S}$ which, when passing the point $B[i]_{L C S}$ through the fibre-processing, characterises the necessary rotation of the frame about an axis head. All the time vector 1 B:array $[i]_{L C S} \perp$ vector 2 B:array $[i]_{L C S}$ holds. We assume that the discrete set of points $B[i]_{L C S}$ lying on the axis $O$ is specified sufficiently densely and defines with a sufficient accuracy the shape of the frame. We suppose the distance of two consecutive points $B[i]_{L C S}$ and $B[i+1]_{L C S}$ has a constant distance $h$. If the polyurethane frame is closed, then the first entered point on the axis $O$ is identical to the last entered point $\left(B[i]_{L C S} \equiv B[N]_{L C S}, \quad\right.$ vector $1 B[i]_{L C S} \equiv$ vector $1 B[N]_{L C S}$ and vector $2 B[i]_{L C S} \equiv$ vector $\left.2 B[N]_{L C S}\right)$. The coordinates of the individual components of the fibre-processing head are entered in the $B C S$ coordinate system. The first outer rotating guide line is presented by the circle $k 1$ with the centre $S 1_{B C S}=\left[x_{S 1}, y_{S 1}, z_{S 1}\right]_{B C S}$, the second outer rotating guide line is presented by the circle $k 2$ with the centre $S 2_{B C S}=\left[x_{S 2}, y_{S 2}, z_{S 2}\right]_{B C S}$, both circles $k 1$ and $k 2$ have the same radius $r_{\text {CIRCLE }}$ (we assume $r_{\text {CIRCLE }}>r_{\text {TUBE }}$ ). Points $S 1$ and $S 2$ lie on the axis $s$ of the fibre-processing head (see Fig. 5). The middle guide line, which enables the placement of the fibres in the longitudinal direction, is static and so it is not necessary to consider this guide line in the model in the subsequent calculations. Point $H_{B C S}$ is entered, this point lies in the middle of the abscissa $S 1_{B C S} S 2_{B C S}$. Subsequently, the unit vectors vector $1 H_{B C S}$ (this vector indicates the direction of passage frame through the fibre-winding head) and vector vector $2 H_{B C S}$ are entered, while the relation vector $1 H_{B C S} \perp$ vector $2 H_{B C S}$ is valid. In our test examples we selected vector $1 H_{B C S}=\left(S 1_{B C S}-S 2_{B C S}\right) /\left\|S 1_{B C S}-S 2_{B C S}\right\|$, where $\left\|S 1_{B C S}-S 2_{B C S}\right\|$ is the length of the abscissa $S 1_{B C S}-S 2_{B C S}$. The point $H_{B C S}$ together with vector $1 H_{B C S}$ and vector $2 H_{B C S}$ allow us to calculate the passage of polyurethane frame through the fibreprocessing head while the frame possibly rotates around the tangent of its axis $o$ in point $B[i]_{B C S}$. In the following chapter we will describe the use of the mathematical model to calculate the trajectory of the robot-end-effector.
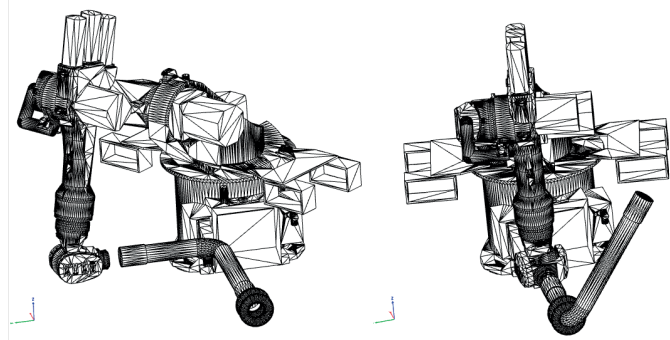

Fig.3 Numerical model describing of fibres winding process for a new composite frame

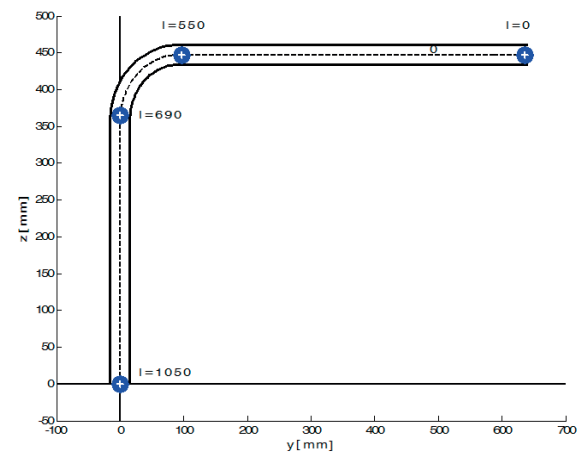

Fig. 4 Geometry of a polyurethane frame

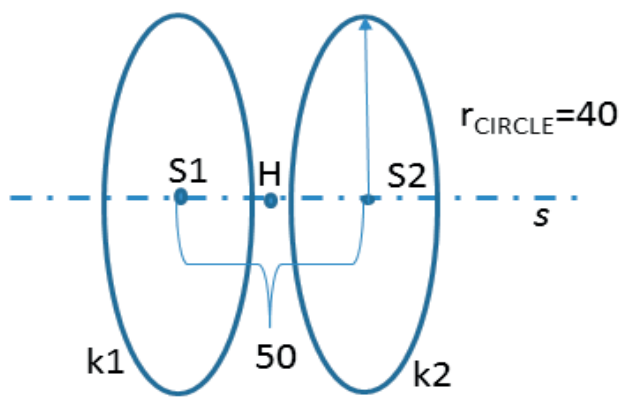

Fig. 5 Points $S 1$ and $S 2$ lie on the axis s of the fibre-processing head 


\subsection{Mathematical model for calculating the trajectory of the robot-end-effector}

In the derivation procedure of trajectory calculation, we used results listed in [1 and 7]. Note that the Denavit-Hartenberg method is often used to determine the trajectory of robot (e.g. see [8]). In this chapter we will calculate such a trajectory of the robot-end-effector when the polyurethane frame attached to the robot-end-effector passes gradually through the fibre-processing head. Recall that the fibre-processing head and its parts are fixedly positioned in the $B C S$ and a position of the polyurethane frame is defined in the LCS of the robot-end-effector. Determination of the trajectory is made on the basis of calculating the sequence of $N$ of the $T C P_{i}$ values, where $1 \leq i \leq N$. Then the robot-end-effector changes its position in relation to changes in parameters based on their linear interpolation (or the use of cubic splines) during the transition from the current $T C P_{i-1}$ to the subsequent $T C P_{i}$ Consequently, the frame passes through the fibre-processing head. The calculated parameters of the current $T C P_{i}$ ensure that point $B[i]_{B C S}$ of the axis $o$ of the frame will be the same as point $H_{B C S}$ (the middle of the fibre-processing head) in the $i$-th step of the polyurethane frame passing through the fibreprocessing head. At the same time the vector vector $1 \mathrm{~B}[i]_{B C S}$ of the tangent of the axis $o$ of the frame at the point $B[i]_{B C S}$ identifies with the vector vector $1 H_{B C S}$ (this vector indicates the direction of passage frame through fibre-winding head) and the vector vector $2 \mathrm{~B}[i]_{B C S}$ identifies with the vector vector $2 H_{B C S}$ (identification of this pair of vectors allows the polyurethane frame to turn around the tangent of the axis of the frame $o$ in the point $\left.B[i]_{B C S}\right)$. When the described identifications are complete (i.e. the two orthogonal vectors and their common starting point entered in the $L C S$ are in the same position in BCS as the two orthogonal vectors and their common starting point in the $B C S$ ) the parameters of the corresponding $T C P_{i}$ are unambiguously determined and, therefore, the position and orientation of the robot-end-effector relative to the $B C S$ in the $i$-th step of passing the frame through the fibre-processing are also unambiguously determined. When calculating the sequence of the $T C P_{i}$ values $(1 \leq i \leq N)$ of the robot-end-effector, we use a matrix calculus to perform a transformation of the $L C S$ of the robot-end-effector relative to the $B C S$. We will write points, vectors and matrices in a homogeneous

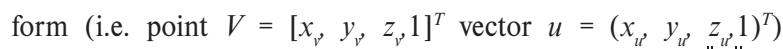
in the following text. We also use the Euclidean norm $\|u\|$ of vector $u$, where $\|u\|=\sqrt{x_{u}^{2}+y_{u}^{2}+z_{u}^{2}}$.

\section{Calculation of the Passage of Frame Thorough the Fibre-processing Head}

We calculate for $i=1, \ldots N$ the $T C P_{i}$ of the robot-end-effector so that at the same time $B[i]_{B C S} \equiv H_{B C S}$, vector $1 B[i]_{B C S} \equiv$ vector $1 H_{B C S}$, vector $2 B[i]_{B C S} \equiv$ vector $2 H_{B C S}$. After determining $T C P_{i}$ all of the $T C P_{i-1}$ parameters are continuously changed to the parameters of to the $T C P_{i}$. By this procedure we determine partial passage of the frame through the fibre-processing head. The initial TCP indicates the position and orientation of the robot-end-effector before the start of the passage of the frame through fibre-processing head. In the following section of this chapter we will focus on the procedure for determining $T C P_{i}$.

\section{Determining the Transformation Matrix}

We assume that $T C P_{i-1}=\left(x_{i-1}, y_{i-1}, z_{i-1}, a_{i-1}, b_{i-1}, c_{i-1}\right)$ is entered. With the aim of determining the transformation matrix $\mathbf{T}_{i}$ (which we will apply to $L C S$ ) in the form Eq. (1) we perform the following steps.

$\mathbf{T}_{i}=\mathbf{L}_{i} \cdot \mathbf{Q}_{i}$

i. We determine the coordinates of the vector vector $1 B[i]_{L C S}$ in the coordinate system $B C S$ (under the assumption that the coordinate systems $B C S$ and $L C S$ have the same origin, this assumption is used when finding the required rotation matrix). The matrix $Q_{i-1}$ of the rotation $L C S$ towards $B C S$ is in the form of Eq. (2).

$Q_{i-1}=\operatorname{Rot}\left(z, a_{i-1}\right) \operatorname{Rot}\left(y, b_{i-1}\right) \operatorname{Rot}\left(x, c_{i-1}\right)$,

where $\operatorname{Rot}\left(z, a_{i-1}\right)$ is the orthogonal matrix of rotation of $L C S$ around axis $z$ at angle $a_{i-1}, \operatorname{Rot}\left(y, b_{i-1}\right)$ orthogonal matrix of rotation of $L C S$ around axis $y$ at angle $b_{i-1}$ and $\operatorname{Rot}\left(x, c_{i-1}\right)$ orthogonal matrix of rotation of $L C S$ around axis $x$ at angle $c_{i-1}(3)$.

$\left.\begin{array}{rl}\operatorname{Rot}\left(z, a_{i-1}\right) & =\left(\begin{array}{cccc}\cos a_{i-1} & -\sin a_{i-1} & 0 & 0 \\ \sin a_{i-1} & \cos a_{i-1} & 0 & 0 \\ 0 & 0 & 1 & 0 \\ 0 & 0 & 0 & 1\end{array}\right) \\ \operatorname{Rot}\left(y, b_{i-1}\right) & =\left(\begin{array}{cccc}\cos b_{i-1} & 0 & \sin b_{i-1} & 0 \\ 0 & 1 & 0 & 0 \\ -\sin b_{i-1} & 0 & \cos b_{i-1} & 0 \\ 0 & 0 & 0 & 1\end{array}\right) \\ \operatorname{Rot}\left(x, c_{i-1}\right) & =\left(\begin{array}{cccc}1 & 0 & 0 & 0 \\ 0 & \cos c_{i-1} & -\sin c_{i-1} & 0 \\ 0 & \sin c_{i-1} & \cos c_{i-1} & 0 \\ 0 & 0 & 0 & 1\end{array}\right)\end{array}\right\}$

Subsequently, we can express the coordinates of the vector vector $1 B[i]_{L C S}$ in the $B C S$ system in the form vector $1 B[i]_{B C S}=$ $Q_{i-1}$ vector $1 B[i]_{L C S}$

ii. By using the scalar product of vectors vector $1 H_{B C S}$ and vector $1 B[i]_{B C S}$ we determine their deviation $\alpha$.

iii. We determine the cross product $p_{B C S}=$ vector $1 H_{B C S} \times$ vector $1 B[i]_{B C S}$.

iv. Vector $p_{B C S}$ is orthogonal to vectors vector $1 H_{B C S}$ and vector $1 B[i]_{B C S^{*}}$.

We normalise vector $p_{B C S}$, i.e., we ensure its unit length $p_{B C S=} p_{B C S} /\left\|p_{B C S}\right\|$. We perform the rotation of vector vector $1 B[i]$ ${ }_{B C S}$ by angle $\alpha$ around vector $p_{B C S}$ (we consider the correct 
$\operatorname{Rot}\left(p_{B C S}, \alpha\right)=\left[\begin{array}{cccc}c+n_{1}^{2}(1-c) & n_{1} n_{2}(1-c)+n_{3} s & n_{1} n_{3}(1-c)-n_{2} s & 0 \\ n_{1} n_{2}(1-c)+n_{3} s & c+n_{2}^{2}(1-c) & n_{2} n_{3}(1-c)-n_{1} s & 0 \\ n_{1} n_{3}(1-c)-n_{2} s & n_{2} n_{3}(1-c)-n_{1} s & c+n_{3}^{2}(1-c) & 0 \\ 0 & 0 & 0 & 1\end{array}\right]$,

orientation of angle vector $1 B[i]_{B C S}$, i.e. rotate vector vector $1 B[i]_{B C S}$ to vector vector $\left.1 H_{B C S}\right)$. Then vector $1 H_{B C S}=\operatorname{Rot}\left(p_{B C S} \alpha\right)$ vector $1 B[i]_{B C S}$ If we denote $p_{B C S}=\left(n_{1}, n_{2}, n_{3}, 0\right)^{T}$, then the matrix $\operatorname{Rot}\left(p_{B C S} \alpha\right)$ will have the form (4), you can see in [1].

Where $s$ and $c$ indicate $s=\sin \alpha, c=\cos \alpha$. At the same time we determine the vector $l_{B C S}(5)$.

$l_{B C S}=\operatorname{Rot}\left(p_{B C S}, \alpha\right) \cdot Q_{i-1}$ vector $2 B[i]_{L C S}$

The cases where $\alpha=0$ or $\alpha=\pi$ need to be solved separately.

v. By using the scalar product of the vectors, we determine the deviation $\beta$ of vectors vector $2 H_{B C S}$ and $l_{B C S^{\circ}}$

vi. The resulting matrix of rotation $Q_{i}$ has the form of Eq. (6).

$$
\begin{aligned}
Q_{i}= & \operatorname{Rot}\left(\operatorname{vector} 1 H_{B C S}, \beta\right) \cdot \operatorname{Rot}\left(p_{B C S}, \alpha\right) \cdot \operatorname{Rot}\left(z, a_{i-1}\right) \cdot \\
& \cdot \operatorname{Rot}\left(y, b_{i-1}\right) \cdot \operatorname{Rot}\left(x, c_{i-1}\right)
\end{aligned}
$$

where the elements of the matrix $\operatorname{Rot}\left(\right.$ vector $\left.1 H_{B C S}, \beta\right)$ are analogously defined as elements of the matrix $\operatorname{Rot}\left(p_{B C S}, \alpha\right)$ in relation (4). In relation (6) we use the correctly determined angle orientation of angle $\beta$, i.e. we rotate the vector $l_{B C S}(5)$ around the vector vector $1 H_{B C S}$ to the vector vector $2 H_{B C S}$.

vii. The translation vector is defined (7) as

$u(i)_{B C S}=H_{B C S}-Q_{i} B[i]_{L C S}-\left(x_{i-1}, y_{i-1}, z_{i-1}, 0\right)^{T}$,

where matrix $Q_{i}$ is determined by the relation (6). We remember that $x_{i-1}, y_{i-1}, z_{i-1}$ are the first three parameters of $T C P_{i-1}$. The resulting transformation matrix $T_{i}$ in the i-th step of the passing of the frame through the fibre-processing head is in the form (8).

$T_{i}=\operatorname{Trans}\left(x_{u(i)}, y_{u(i)}, z_{u(i)}\right) \cdot Q_{i}$,

where translation matrix

$\operatorname{Trans}\left(x_{u(i)}, y_{u(i)}, z_{u(i)}\right)=\left(\begin{array}{cccc}1 & 0 & 0 & x_{u(i)} \\ 0 & 1 & 0 & y_{u(i)} \\ 0 & 0 & 1 & z_{u(i)} \\ 0 & 0 & 0 & 1\end{array}\right)$

and $x_{u(i)}, y_{u(i)}, z_{u(i)}$ are components of vector $u(i)_{B C S}$ in Equation (7).

After performing the LCS of the robot-end-effector the corresponding transformation matrix $T_{i}$ determined by relation (8) will be valid $H_{B C S} \equiv B[i]_{B C S}=T_{i} B[i]_{L C S}$ and vector $1 B[i]_{B C S} \equiv$ vector $1 H_{B C S}$, vector $2 B[i]_{B C S} \equiv$ vector $2 H_{B C S}$.

\section{III.Calculation of the Euler Angles}

We will calculate the Euler angles. Any right-handed rotation in Euclidean space $\mathrm{E}_{3}$ around the given unit vector $p$ by angle $\varphi$ is determined by the orthogonal matrix $\mathbf{Q}=\operatorname{Rot}(\mathbf{p}, \varphi)$, its elements are in the form ( 3 ) and $\operatorname{det} \mathbf{Q}=1$. Each rotation matrix can be written as a product of the matrices of rotation around the coordinate axes $z, y$ and $x$, i.e. $Q=\operatorname{Rot}(z, a) \cdot \operatorname{Rot}(y, b) \cdot \operatorname{Rot}(x, c)$, where the matrices $\operatorname{Rot}(z, a), \operatorname{Rot}(y, b)$ and $\operatorname{Rot}(x, c)$ are in the form (3); $a, b$ and $c$ are the corresponding Euler angles. We note that the determination of Euler angles $a, b$ and $c$ is not unique (see [7]). The matrix of rotation $Q_{i}$ defined by the relation (9) can be decomposed into the product of the orthogonal matrices.

$Q_{i}=\operatorname{Rot}\left(z, a_{i}\right) \cdot \operatorname{Rot}\left(y, b_{i}\right) \cdot \operatorname{Rot}\left(x, c_{i}\right)$

By multiplying the left and right sides from the left of the matrix $\operatorname{Rot}\left(z, a_{i}\right)^{-1}$ because the matrix $\operatorname{Rot}\left(z, a_{i}\right)$ is orthogonal $\left.\operatorname{Rot}\left(z, a_{i}\right)^{-1}=\operatorname{Rot}\left(z, a_{i}\right)^{T}\right)$ and comparing suitably selected corresponding elements from the resulting matrix on the left and right sides of equation (8) modified in this way, we can determine the rotation angles $a_{i}, b_{i}$ and $c_{i}$ (see [4], pp. 32). When calculating the angles of rotations, we use the ATAN2 function (part of the library of most programming languages), which calculates from the two input parameters $\arg _{1}$ and $\arg _{2}$ the value of the function arctangent for argument $\arg _{1} / \arg _{2}$. Moreover, the signs of both input parameters are used to determine the quadrant in which the resulting value function is located (it is valid that $-\pi<\operatorname{ATAN2}\left(\arg _{1}\right.$, $\left.\arg _{2}\right) \leq \pi$ part). We write the matrix of rotation $Q_{i}$ in the form by (10).

$Q_{i}=\left(\begin{array}{cccc}q_{11}(i) & q_{12}(i) & q_{13}(i) & 0 \\ q_{21}(i) & q_{22}(i) & q_{23}(i) & 0 \\ q_{31}(i) & q_{32}(i) & q_{33}(i) & 0 \\ 0 & 0 & 0 & 1\end{array}\right)$

Using the procedure described above for comparing the corresponding elements of the matrices in the modified equation (9) it is possible to obtain (11).

$$
\left.\begin{array}{l}
a_{i}=\operatorname{ATAN2}\left(q_{21}(i), q_{11}(i)\right), \\
b_{i}=\operatorname{ATAN2}\left(\begin{array}{l}
-q_{31}(i), q_{11}(i) \cos a_{1}+q_{21} \\
(i) \sin a_{1}
\end{array}\right), \\
c_{i}=\operatorname{ATAN2}\left(\begin{array}{l}
q_{13}(i) \sin a_{i}-q_{23}(i) \cos a_{i} \\
q_{22}(i) \cos a_{1}-q_{12} \sin a_{i}
\end{array}\right)
\end{array}\right\}
$$


Thus, we determined rotation angles $a_{i}, b_{i}, c_{i}$ in the equation (9) and the corresponding $T C P_{i}$ defining the position and orientation of the $L C S$ of the robot-end-effector in relation to the $B C S$ of the robot. The $T C P_{i}$ can be expressed in the form (12).

$T C P_{i}=\left(x_{u(i)}, y_{u(i)}, z_{u(i)}, a_{i}, b_{i}, b_{i}\right)$

where the elements of the vector $u(i)_{B C S}$ are given by the relation (7).

\section{Results and discussion}

We focus on the practical problem of the passage of the non-bearing polyurethane frame with a circular cross-section through the fibre-processing head. The central 2D axis o of the frame is composed of two interconnected perpendicular arms (see Fig. 4). The axis $o$ is entered into the $L C S$ of the robot-end-effector using a discrete set of points $B[i]_{L C S}$, $1 \leq i \leq N=105$. The total length $d$ of the axis $o$ from the starting point $B[1]_{L C S}$ to the end point $B[N]_{L C S}$ of the axis is $d$ $=1050$. The continuous distance of the points $B[i]_{L C S}$ on the axis $o$ from the starting point is denoted by the variable $l$. The distance between two consecutive points $B[i]_{L C S}$ and $B[i+1]_{L C S}$ is $h=10 \mathrm{~mm}$. Vectors vector $1 B[i]_{L C S}$ and vector $2 B[i]_{L C S}$ are specified, radius of the frame is $r_{\text {TUBE }}=20 \mathrm{~mm}$. The fibre-processing head is represented by the circles $k 1$ and $k 2$ with the centres $S 1_{B C S}=[-1000,1105,990]$ and $S 2_{B C S}=[-1000,1035,990]$ having the same radius $r_{\text {CIRCLE }}=40 \mathrm{MM}$. The length of abscissa $S 1_{B C S} S 2_{B C S}=70 \mathrm{~mm}$. It is valid $H_{B C S}=\left(S 1_{B C S}+S 2_{B C S}\right) / 2$, vector $1 H_{B C S}=\left(S 1_{B C S}-S 2_{B C S}\right) /\left\|S 1_{B C S}-S 2_{B C S}\right\|$, vector $2 H_{B C S}=(0,0,1)$. The frame needs to be rotated at a distance of $550 \mathrm{~mm}$ from the beginning of the axis $o$ to the distance of $690 \mathrm{~mm}$ around a tangent to the frame of axis $o$ during the passing of point $B[i]_{B C S}$ (for $56 \leq i \leq 69$ ) by point $H_{B C S}$ when placing the longitudinal fibres relative to the axis $o$ of the frame. This is a uniform right-handed rotary motion with an overall rotation angle $\omega=\pi$. The rotation is performed during the passage of the bent portion of the frame through the fibre-processing head. The calculation of the trajectory of the robot-end-effector from numerical model referred to in the previous chapter was applied to the described problem. Fig. 6 shows the position of the robot-end-effector when passing the polyurethane frame through winding head. Part a) shows the position of the robot-end-effector (first three parameters of $T C P$ ) and part b) the orientation of the robot-end-effector (last three parameters of $T C P$ ). Figures 7 and 8 compare the numerical model and the real experiment and illustrate the individually calculated values of $T C P$ during the passing of the frame through the fibre-processing head. The comparison of an ideal sample and real sample from winding processing is seen in Fig. 9. Our article solves problem in which a composite frame with a circular cross-section is attached to

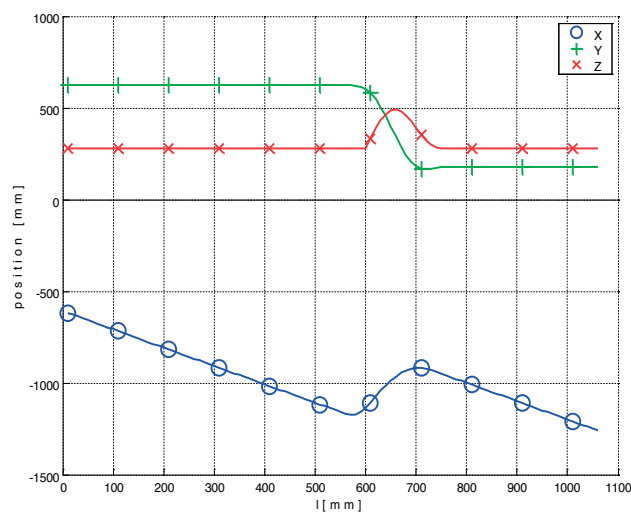

a)

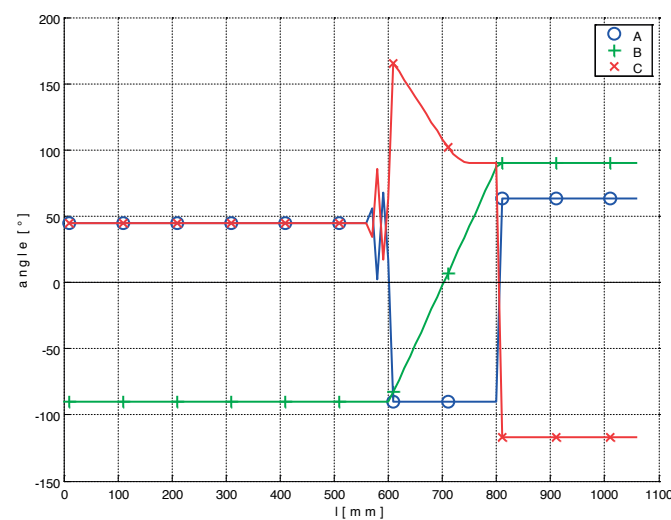

b)

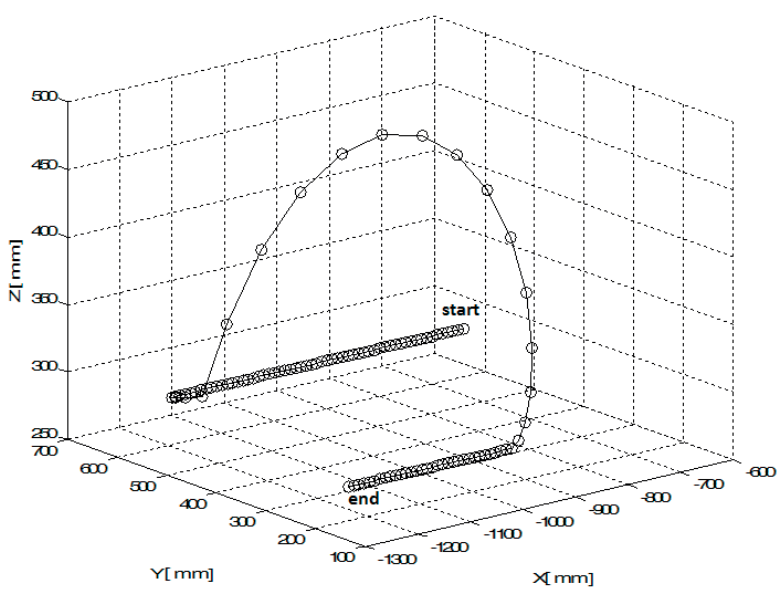

c)

Fig. 6 Diagram showing numerical simulation of the course of the TCP during the passing of the frame through the fibre-processing head, a) parameter values of the first three parameters of TCP, b) parameter values of the last three parameters of TCP, c) trajectory of the robot-end-effector: start and end 

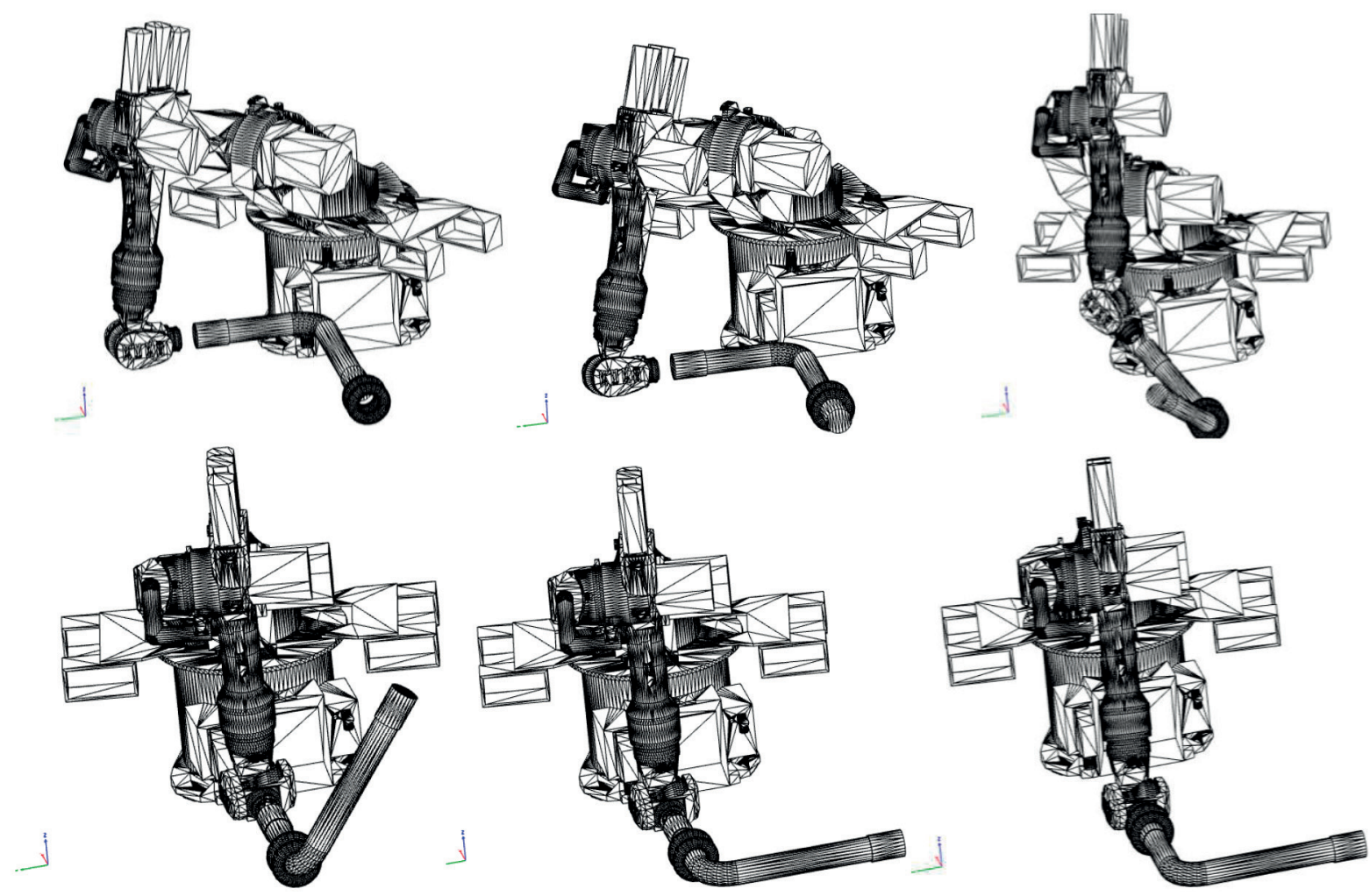

Fig. 7 Time response of a numerical model for optimal trajectory of fibre winding process

the end-effector of the industrial robot and successively passes through the fibre-processing head during the winding process. It is a similar problem as in [1 and 7]. We don't know any other
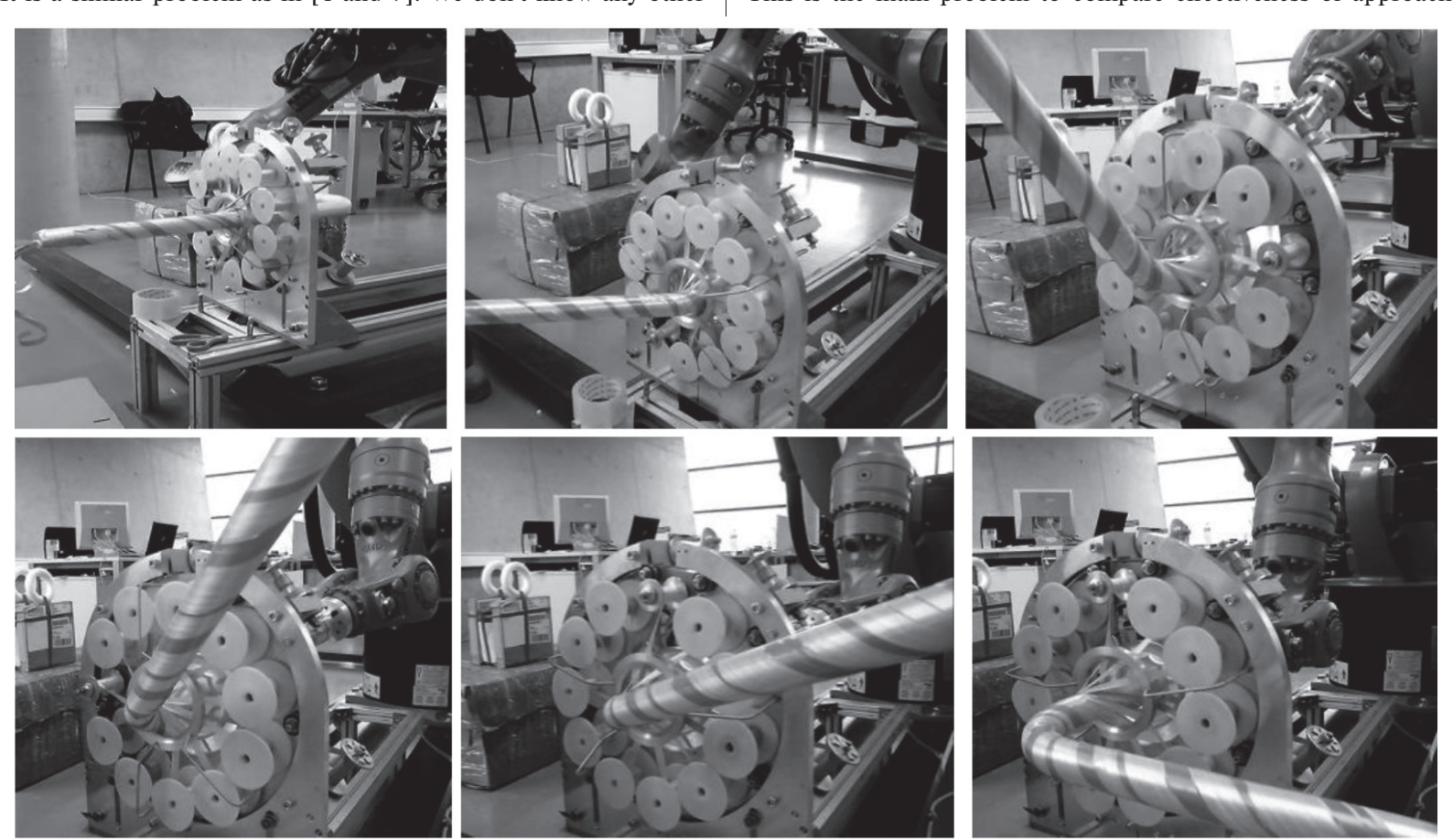

robotic workplace where similar technical problem production of composite with frame shape (the frame could be closed) is solved. This is the main problem to compare effectiveness of approach

Fig. 8 Time response of real fibre winding process with data from a numerical model 

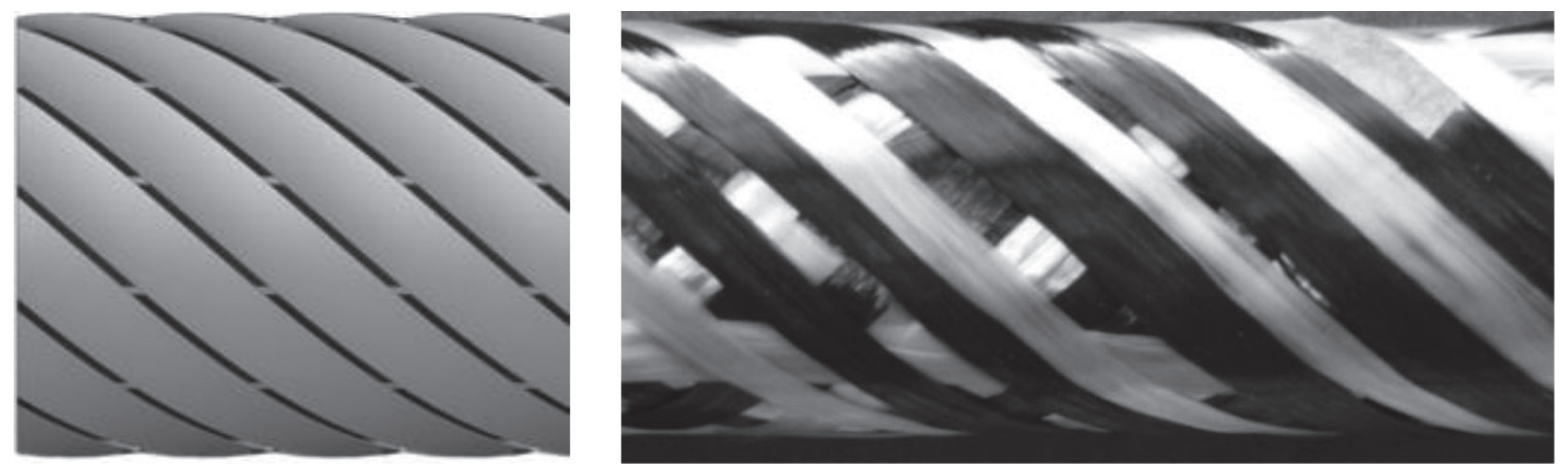

Fig. 9 Comparison an ideal sample and real sample from winding processing with data from a numerical model

of our production procedure with the other one. We solved determining of the robot-end-effector trajectory by our procedure in several practical examples and we have obtained good results. Of course in the world you can find a number of professional teams that are trying to find an optimal algorithm for computing the trajectory toward solving a particular problem. All these methods are, but intended for surface coating technology fibres. Therefore not directly comparable with the method presented in this article (which is designed for winding fibres). Their basic aim is that the product (its surface) is placed firmly in the space robot and carries a tool for applying fibres. They are known methods such as point Tag generation for flat open surfaces [9], where it is the automatic generation of points based on a CAD product model and tools. Fibre steering is method for determining the trajectory of the robot, the robot gives fibres along a circular path on a flat or complex surface (helix) [9]. Surface-curve algorithm for RFP (SCAR), where is the basic principle of this method is defining an initial curve for laying fibres to form [10]. Roller path planning method is the method similar to method RFP (SCAR), but examines not only the position of the fibre to be laid, but also the availability of trajectory from a pressure roller [11].

\section{Conclusion}

The described mathematical or numerical model with algorithm allows the accurate calculation and determination of the $3 \mathrm{D}$ trajectory of the robot-end-effector of the industry robot during the production of composite profile using the dry fibre winding technology on a open and closed non-bearing frame. This provides a significant advantage over users of the extended teach-in principle. The determination of the trajectory of the robot-end-effector using the given method can be used for optimising the trajectory of the robot-end-effector, too. Evolutionary optimising algorithms, in particular genetic algorithms or differential algorithms are often used in solving technical problems (see e.g. [6 - 8]). The described algorithm trajectory calculation can be applied to any manufacturing process where it is necessary to determine the $3 \mathrm{D}$ trajectory of a robotend-effector. Suppliers of industrial robots currently offer specific software tools facilitating control of the robot-end-effector when programming specific tasks (e.g. welding, pressing, laser cutting, grinding). However, none of them are suitable to solve a technical problem of composite production and other general tasks related to the use of industrial robots. The use of the described algorithm is completely independent on the type of industrial robot and software tools. The procedure for determining the trajectory of the robot-end-effector induces virtually no additional costs to the manufacturer and can significantly speed up the determination of the desired trajectory of the robot-end-effector.

\section{Acknowledgements}

The results of this project No. LO1201 were obtained through the financial support of the Ministry of Education, Youth and Sports, Czech Republic in the framework of the targeted support of the "National Programme for Sustainability I".

\section{References}

[1] PETRU, M., MARTINEC, T., MLYNEK, J.: Numerical Model Description of Fibres Winding Process for new Technology of Winding Fibres on the Frames, Manufacturing Technology, vol.16, No. 4, pp. 778-785, 2016.

[2] NOVAKOVA-MARCINCINOVA, L., NOVAK-MARCINCIN, J.: Production of ABS-aramid Composite Material by Fused Deposition Modeling Rapid Prototyping System. Manufacturing Technology, vol. 14, No. 1, pp. 85-91, 2014. 
[3] PETRU, M., BRONCEK, J., LEPSIK, P., NOVAK, O.: Experimental and Numerical Analysis of Crack Propagation on Light Composite Materials under Dynamic Fracturing, Communications - Scientific Letters of the University of Zilina, vol. 16, No. 3A, pp. 82-89, 2014.

[4] SCIAVICCO, L., SICILIANO, B.: Modelling and Control of Robot Manipulators, p. 378, 2004, Springer : London.

[5] JAZAR R., N.: Theory and Applied Robotics, p. 883, 2004, Springer : New York.

[6] SCIAVICCO, L., SICILIANO, B.: Modelling and Control of Robot Manipulators, p. 378, 2004, Springer : London.

[7] MARTINEC, T., MLYNEK, J., PETRU, M.: Calculation of the Robot Trajectory for the Optimum Directional Orientation of Fibre Placement in the Manufacture of Composite Profile Frames, Robotics and Computer-Integrated Manufacturing, vol. 35, pp. 42-54, 2015.

[8] BALOCKOVA, L.: The Method for Solving Kinematics of an Industrial Robot, Applied Mechanics and Materials, vol. 283, pp. 279281, 2013.

[9] SHIRINZADECH, B., ALICI, G., FOONG, C. W., CASSIDY, G.: Fabrication Process of Open Surfaces by Robotic Fibre Placement. Robotics and Computer-Integrated Manufacturing, vol. 20, pp. 17-28, 2004.

[10] SHIRINZADECH, B., CASSIDY, G., OETOMO, D., ALICI, G., ANG, M. H.: Trajectory Generation for Open-Contoured Structures, Robotic Fibre Placement. Robotics and Computer-Integrated Manufacturing, vol. 23, pp. 276-286, 2007.

[11] LONG, Y., ZEZHONG, CH. CH., YAOYAO, S., RONG, M.: An Accurate Approach to Roller Path Generation for Robotic Fibre Placement of Free-Form Surface Composites. Robotics and Computer-Integrated Manufacturing, vol. 30, No. 3, pp. 277-286, 2014. 